\title{
Recent Advances on Boundary Conditions for Equations in Nonequilibrium Thermodynamics
}

\author{
Wen-An Yong ${ }^{1,2, *}$ and Yizhou Zhou ${ }^{3}$ \\ 1 Department of Mathematical Sciences, Tsinghua University, Beijing 100084, China \\ 2 Yanqi Lake Beijing Institute of Mathematical Sciences and Applications, Beijing 101408, China \\ 3 School of Mathematical Sciences, Peking University, Beijing 100871, China; zhouyz@math.pku.edu.cn \\ * Correspondence: wayong@tsinghua.edu.cn
}

check for

updates

Citation: Yong, W.-A.; Zhou, Y. Recent Advances on Boundary Conditions for Equations in Nonequilibrium Thermodynamics. Symmetry 2021, 13, 1710. https:/ / doi.org/10.3390/sym13091710

Academic Editors: Róbert Kovács, Patrizia Rogolino, Francesco Oliveri, Charles F. Dunklm, Marin Marin and Mariano Torrisi

Received: 10 May 2021

Accepted: 1 September 2021

Published: 16 September 2021

Publisher's Note: MDPI stays neutral with regard to jurisdictional claims in published maps and institutional affiliations.

\begin{abstract}
This paper is concerned with modeling nonequilibrium phenomena in spatial domains with boundaries. The resultant models consist of hyperbolic systems of first-order partial differential equations with boundary conditions (BCs). Taking a linearized moment closure system as an example, we show that the structural stability condition and the uniform Kreiss condition do not automatically guarantee the compatibility of the models with the corresponding classical models. This motivated the generalized Kreiss condition (GKC) - a strengthened version of the uniform Kreiss condition. Under the GKC and the structural stability condition, we show how to derive the reduced BCs for the equilibrium systems as the classical models. For linearized problems, the validity of the reduced BCs can be rigorously verified. Furthermore, we use a simple example to show how thus far developed theory can be used to construct proper BCs for equations modeling nonequilibrium phenomena in spatial domains with boundaries.
\end{abstract}

Keywords: hyperbolic relaxation system; structural stability condition; generalized Kreiss condition

\section{Introduction}

Irreversible thermodynamics is a theory in physics for the mathematical modeling of nonequilibrium processes. The resultant models are usually time-dependent partial differential equations (PDEs) [1]. So far, the theory is still developing, and there are no well-accepted rules in establishing the equations. Therefore a number of different theories exist [2-8], leading to various PDEs. It is a challenging issue to evaluate the reasonableness of the different equations. To do so, four fundamental requirements were proposed and expounded in [9]. They are the observability of physical phenomena, time irreversibility, long-time tendency to equilibrium, and compatibility with possibly existing classical theories.

Recall well-known theories extended irreversible thermodynamics (EIT) [4,5]), rational extended thermodynamics (RET) [6]), and conservation-dissipation formalism (CDF) [8]). Irreversible processes can be described with PDEs of the form:

$$
U_{t}+\sum_{j=1}^{d} F_{j}(U)_{x_{j}}=\tilde{Q}(U)
$$

Here, $U=U(x, t) \in G \subset \mathbb{R}^{n}$ is the unknown function of $x=\left(x_{1}, x_{2}, x_{d}\right)$ with $d(=1,2$ or 3$)$ for the spatial dimension and $t>0, G$ is an open set called the state space, $F_{j}(U)$ is the flux along the $x_{j}$-direction, source term $\tilde{Q}(U)$ represents the thermodynamical force, and subscripts $t, x_{j}$ denote partial derivatives with respect to the corresponding variables. Very often, the source term can be written as

$$
\tilde{Q}(U)=\left(\begin{array}{c}
0 \\
q(U)
\end{array}\right) .
$$


Accordingly, we partition

$$
U=\left(\begin{array}{c}
u \\
v
\end{array}\right), \quad F_{j}(U)=\left(\begin{array}{l}
f_{j}(u, v) \\
g_{j}(u, v)
\end{array}\right) .
$$

With this partition, $u \in \mathbb{R}^{n-r}$ stands for the conserved variables, and $v \in \mathbb{R}^{r}$ is referred to as nonequilibrium variables. To cover more equations from EIT $[4,5]$ and general equation for nonequilibrium reversible-irreversible coupling (GENERIC) [7,10]), we also consider the following equation.

$$
U_{t}+\sum_{j=1}^{d} A_{j}(U) U_{x_{j}}=\tilde{Q}(U),
$$

which is more general than (1). Indeed, (1) can be written as (2) with $A_{j}(U)=\frac{\partial F_{j}}{\partial U}(U)$.

For such first-order PDEs, the first fundamental requirement corresponds to the hyperbolicity of Equation (2) [11,12]. To see the other requirements, thermodynamic force $Q(U)$ contains a relaxation time $\epsilon$ in general, and we explicitly write it as $\tilde{Q}(U)=Q(U) / \epsilon$. The requirements are closely related to the limit as the relaxation time $\epsilon$ tends to zerothe so-called zero relaxation limit. Without considering the boundary conditions, the four requirements are fulfilled by (2) if the PDEs satisfy the structural stability condition proposed in [13,14]. See [14,15].

When the nonequilibrium phenomena occur in a spatial domain with boundaries, the PDEs alone cannot completely describe the physical processes, and proper boundary conditions (BCs) are indispensable. However, it is a challenging task to have such $\mathrm{BCs}$, since the physical meaning of the nonequilibrium variables is often unclear (e.g., the higher-order moments in moment-closure systems $[6,16])$. On the other hand, formulating proper BCs for hyperbolic systems of PDEs is mathematically a tough issue [17]. For example, the number of $\mathrm{BC}$ s for (2) should be equal to the number of positive eigenvalues of coefficient matrix $A_{1}(U)$ in case that the boundary is $x_{1}=0$; moreover, the BCs should satisfy the uniform Kreiss condition (UKC) [18] to ensure the well-posedness of the complete model (PDEs together with $\mathrm{BCs}$ ) - the first fundamental requirement. Furthermore, the rest requirements also apply to the complete model. This suggests to study the zero relaxation limit of initial-boundary-value problems (IBVPs) for hyperbolic systems (1) or (2). The resultant results are expected to be useful in providing reasonable constraints for the possible BCs and in developing a systematical method to construct the required BCs.

The paper presents the first author's considerations in the past two decades and recent results on these issues. It is organized as follows. Section 2 contains some basic knowledges about relaxation system (3) and boundary conditions. In Section 3, we show that the structural stability condition and the UKC do not automatically guarantee compatibility, and present a strengthened version of the UKC — the generalized Kreiss condition (GKC). Section 4 is devoted to the derivation of the boundary condition for the zero relaxation limit under the GKC and the structural stability condition. In Section 5, we use an example to show how the theory presented above can be used to construct BCs for the PDEs (3). Some concluding remarks are given in Section 6.

\section{Preliminaries}

To address the long-term tendency and compatibility requirements, we explicitly introduce relaxation time $\epsilon$ and write Equation (3) as

$$
U_{t}+\sum_{j=1}^{d} A_{j}(U) U_{x_{j}}=\frac{1}{\epsilon} Q(U)
$$

For such a small parameter problem, the most fundamental is the following structural stability condition proposed in $[13,14]$ : 
(i) There is an invertible $n \times n$-matrix $P(U)$ and an invertible $r \times r$-matrix $S(U)(0<r \leq n)$, defined on equilibrium manifold $\mathcal{E}=\{U \in G: Q(U)=0\}$, such that

$$
P(U) Q_{U}(U)=\left(\begin{array}{cc}
0 & 0 \\
0 & S(U)
\end{array}\right) P(U) \quad \text { for } U \in \mathcal{E} ;
$$

(ii) As a hyperbolic system, (1) is symmetrizable, that is, there is a positive definite symmetric matrix $A_{0}(U)$, such that

$$
A_{0}(U) A_{j}(U)=A_{j}^{*}(U) A_{0}(U) \text { for } U \in G ;
$$

(iii) The hyperbolic part and the source term are coupled in the following sense.

$$
A_{0}(U) Q_{U}(U)+Q_{U}^{*}(U) A_{0}(U) \leq-P^{*}(U)\left(\begin{array}{cc}
0 & 0 \\
0 & I_{r}
\end{array}\right) P(U) \quad \text { for } U \in \mathcal{E} .
$$

Here and below, $Q_{U}$ denotes the Jacobian matrix of $Q=Q(U), I_{k}$ denotes the unit matrix of order $k$, and superscript $*$ denotes the (conjugate) transpose of a matrix.

In the structural stability condition, (i) is just the usual assumption in the corresponding theory for ordinary differential equations [14], that is, only for the source term; (ii) is for the part of spatial derivatives and means that (3) is symmetrizable hyperbolic; and (iii) is a coupling condition that involves the both parts. As observed in [14,19], the structural stability condition is fulfilled by many classical models from mathematical physics. It is related to the Onsager reciprocal relation and can be viewed as a stability criterion for nonequilibrium thermodynamics. Regarding the Onsager relation, matrix $A_{0}(U) Q_{U}(U)$ is often symmetric. For simplicity, this symmetry is assumed throughout the paper.

Under the structural stability condition, it was proved in $[13,14]$ that the solution to the initial-value problem of (3) converges to that of the corresponding equilibrium system as $\epsilon$ goes to zero. For the case where

$$
Q(U)=\left(\begin{array}{c}
0 \\
q(U)
\end{array}\right)
$$

with $q=q(U) \in \mathbb{R}^{r}$, we write

$$
U=\left(\begin{array}{c}
u \\
v
\end{array}\right), \quad A_{j}(U)=\left(\begin{array}{cc}
A_{j 11} & A_{j 12} \\
A_{j 21} & A_{j 22}
\end{array}\right)(U) .
$$

In this case, it is reasonable to assume that $q(U)=0$ if and only if $v=h(u)$. Then, the equilibrium system can be expressed as

$$
\begin{gathered}
v=h(u), \\
u_{t}+\sum_{j=1}^{d} A_{j 11}(u, h(u)) u_{x_{j}}+\sum_{j=1}^{d} A_{j 12}(u, h(u)) h(u)_{x_{j}}=0 .
\end{gathered}
$$

Under the structural stability condition, it was also proved in $[13,20]$ that the equilibrium system is symmetrizable hyperbolic.

When Equation (3) is given in a spatial domain with boundaries, proper boundary conditions (BCs) should be prescribed to solve the equations. By referring to [21], it is adequate to consider that the spatial domain is half-space

$$
\left(x_{1}, x_{2}, \ldots, x_{d}\right) \equiv\left(x_{1}, \hat{x}\right) \in[0,+\infty) \times \mathbb{R}^{d-1} .
$$


For this domain, assume that coefficient matrix $A_{1}(U)$ has $p$ positive eigenvalues. According to the classical theory of hyperbolic equations $[17,22], p$ independent $B C s$

$$
B(U(0, \hat{x}, t) ; t)=0
$$

should be prescribed at boundary $x_{1}=0$. Moreover, they should satisfy the uniform Kreiss condition (UKC) [18] to ensure the well-posedness, namely, there is a positive constant $c_{K}$, such that

$$
\left|\operatorname{det}\left\{B_{U} R_{\tilde{M}}^{S}(\xi, \omega)\right\}\right| \geq c_{K} \sqrt{\operatorname{det}\left\{R_{\tilde{M}}^{S *}(\xi, \omega) R_{\tilde{M}}^{S}(\xi, \omega)\right\}}
$$

for all $\omega \in \mathbb{R}^{d-1}$ and all complex number $\xi$ with $\operatorname{Re} \xi>0$. To decode this UKC, we assume that $A_{1}(U)$ is invertible, and know from [18] that matrix

$$
\tilde{M}(\xi, \omega):=A_{1}^{-1}\left(-\xi I+i \sum_{j=2}^{d} \omega_{j} A_{j}\right)
$$

has $p$ stable eigenvalues (eigenvalues with negative real parts) for $\operatorname{Re} \xi>0$ and $\omega=\left(\omega_{2}, \ldots, \omega_{d}\right) \in \mathbb{R}^{d-1}$. Then, there is a full-rank $n \times p$-matrix $R_{\tilde{M}}^{S}(\xi, \omega)$ (so-called rightstable matrix of $\tilde{M}[23])$ satisfying

$$
\tilde{M}(\xi, \omega) R_{\tilde{M}}^{S}(\xi, \omega)=R_{\tilde{M}}^{S}(\xi, \omega) \tilde{\Lambda}(\xi, \omega)
$$

with $\tilde{\Lambda}(\xi, \omega)$ a $p \times p$-matrix having $p$ stable eigenvalues.

When $A_{1}(U)$ is not invertible, boundary $x_{1}=0$ is referred to as characteristic. In this case, a modified UKC was formulated in [21] as a sufficient and essentially necessary condition for well-posedness. In addition, it was indicated in [21] that the boundary condition should not involve characteristic modes corresponding to the zero eigenvalue.

\section{Generalized Kreiss Condition}

This section shows that the structural stability condition together with the UKC do not automatically guarantee a well-behaved zero relaxation limit. To do this, we start with the linearized version of PDEs in (3):

$$
U_{t}+\sum_{j=1}^{d} A_{j} U_{x_{j}}=\frac{1}{\epsilon} Q U,
$$

together with homogeneous boundary conditions

$$
B U(0, \hat{x}, t)=0
$$

for $x=\left(x_{1}, \hat{x}\right)=\left(x_{1}, x_{2}, \ldots, x_{d}\right)$ with $x_{1}>0$. Here, $A_{j}(j=1,2, \ldots, d)$ and $Q$ are $n \times n$ constant matrices and $B$ is a full-rank constant $p \times n$-matrix with $p$ the number of positive eigenvalues of $A_{1}$.

Following $[18,23,24]$, we consider the corresponding eigenvalue problem:

$$
\left\{\begin{array}{l}
\xi \hat{U}+A_{1} \hat{U}_{x_{1}}-i \sum_{j=2}^{d} \omega_{j} A_{j} \hat{U}=Q \hat{U}, \\
B \hat{U}(0)=0
\end{array}\right.
$$

for complex number $\xi$ with $\operatorname{Re} \xi>0$ and $\omega=\left(\omega_{2}, \ldots, \omega_{d}\right) \in \mathbb{R}^{d-1}$. Let $\hat{U}=\hat{U}\left(x_{1}\right)$ be a bounded solution to the above problem. It is not difficult to verify that

$$
U^{\epsilon}(x, t):=\exp \left(\frac{\xi t}{\epsilon}-\frac{i \omega \cdot \hat{x}}{\epsilon}\right) \hat{U}\left(\frac{x_{1}}{\epsilon}\right)
$$


solves (5) and (6) with a bounded initial value. Since $\operatorname{Re} \xi>0$, such a solution exponentially increases as $\epsilon$ goes to zero for any $t>0$. Apparently, the zero relaxation limit is not well-behaved or does not exist in this situation.

In order to see when problem (7) has bounded solutions, we assume $A_{1}$ to be invertible and rewrite (7) as

$$
\frac{d \hat{U}}{d x_{1}}=M(\xi, \omega, 1) \hat{U}
$$

with

$$
M(\xi, \omega, \eta)=A_{1}^{-1}\left(\eta Q-\xi I+i \sum_{j=2}^{d} \omega_{j} A_{j}\right) .
$$

Here, $\eta \geq 0$ was introduced to cover the case without source term $Q$, namely, $M(\xi, \omega, 0)=\tilde{M}(\xi, \omega)$ in Section 2 .

According to Lemma 2.3 in [23], under the structural stability condition, matrix $M=M(\xi, \omega, \eta)$ has precisely $p$ stable eigenvalues (with negative real parts) and $(n-p)$ unstable eigenvalues. Then, there is a full-rank $n \times p$-matrix $R_{M}^{S}=R_{M}^{S}(\xi, \omega, \eta)$ satisfying

$$
M R_{M}^{S}=R_{M}^{S} \Lambda
$$

with $\Lambda$ a $p \times p$-matrix having $p$ stable eigenvalues. If the $p \times p$-matrix $B R_{M}^{S}(\xi, \omega, 1)$ is not invertible, then there is a nonzero $p$-vector $\zeta$, such that $B R_{M}^{S}(\xi, \omega, 1) \zeta=0$. With $\hat{U}(0)=$ $R_{M}^{S}(\xi, \omega, 1) \zeta$ as initial value, the ordinary differential Equation (8) has a bounded solution for $x_{1}>0$.

Consequently, we obtain

Proposition 1. If there exists $\xi$ with $\operatorname{Re} \xi>0$ such that the $p \times p$-matrix $B R_{M}^{S}(\xi, \omega, 1)$ is singular, then the problem in (5) and (6), with a bounded initial value, admits an exponentially increasing solution for $t>0$ as $\epsilon$ goes to zero.

The above discussion can be illustrated with the one-dimensional linearized moment closure system [16,25]:

$$
\left\{\begin{array}{l}
U_{t}+A_{1} U_{x}=Q U / \epsilon, \\
B U(0, t)=0
\end{array}\right.
$$

with $Q=\operatorname{diag}(0,0,0,-1)$ and

$$
A_{1}=\left(\begin{array}{cccc}
0 & \rho_{0} & 0 & 0 \\
\theta_{0} / \rho_{0} & 0 & 1 & 0 \\
0 & 2 \theta_{0} & 0 & 6 / \rho_{0} \\
0 & 0 & \rho_{0} \theta_{0} / 2 & 0
\end{array}\right), \quad B=\left(\begin{array}{cccc}
0 & 1 & 0 & 0 \\
0 & 0 & \chi \rho_{0} \sqrt{\theta_{0}} & -1
\end{array}\right) .
$$

Here, $U=(\rho, u, \theta, f)$ is the unknown. $\rho, u, \theta$ are fluid density, velocity, and temperature, respectively, while $f$ is related to the heat flux. Constants $\rho_{0}$ and $\theta_{0}$ in coefficient matrix $A_{1}$ denote density and temperature at the equilibrium. Constant $\chi$ in the $\mathrm{BC}$ is a positive parameter. Obviously, the moment-closure system satisfies the structural stability condition with symmetrizer

$$
A_{0}=\left(\begin{array}{cccc}
2 \theta_{0}^{3} & 0 & 0 & 0 \\
0 & 2 \rho_{0}^{2} \theta_{0}^{2} & 0 & 0 \\
0 & 0 & \rho_{0}^{2} \theta_{0} & 0 \\
0 & 0 & 0 & 12
\end{array}\right)
$$


It was shown in [25] that the BC in (9) satisfies the UKC if and only if

$$
x \neq \frac{\sqrt{3+\sqrt{6}}+\sqrt{3-\sqrt{6}}}{2(3+\sqrt{3})} .
$$

Moreover, Proposition 3.3 in [25] says that if

$$
x \in\left(0, \frac{\sqrt{3+\sqrt{6}}+\sqrt{3-\sqrt{6}}}{2(3+\sqrt{3})}\right)
$$

there is a positive $\xi$ such that $B R_{M}^{S}(1, \xi)$ is singular. According to Proposition 1, the structural stability condition and the UKC do not automatically imply the existence of the zero relaxation limit.

Proposition 1 and the example above indicate the following necessary condition for the existence of zero relaxation limit:

$$
\operatorname{det}\left\{B R_{M}^{S}(\xi, \omega, 1)\right\} \neq 0, \text { for any } \operatorname{Re} \xi>0, \omega \in \mathbb{R}^{d-1} .
$$

Motivated by this, the following condition was proposed in [23]:

Generalized Kreiss Condition. There exists a constant $c_{K}>0$, such that

$$
\left|\operatorname{det}\left\{B R_{M}^{S}(\xi, \omega, \eta)\right\}\right| \geq c_{K} \sqrt{\operatorname{det}\left\{R_{M}^{S *}(\xi, \omega, \eta) R_{M}^{S}(\xi, \omega, \eta)\right\}}
$$

for all $\eta \geq 0, \omega \in \mathbb{R}^{d-1}$ and $\xi$ with $\operatorname{Re} \xi>0$.

Clearly, this GKC does not depend on the special choice of $R_{M}^{S}(\xi, \omega, \eta)$. It recovers the standard UKC when $\eta=0$. Unlike the UKC, the GKC involves parameters even for one-dimensional problems. Moreover, it was shown in [23] that the GKC holds if the BCs are strictly dissipative in the following sense:

Definition 1. The boundary condition is referred to as strictly dissipative with respect to symmetrizer $A_{0}$ and $A_{1}$, if there is a positive constant $c_{d}$, such that

$$
y^{*} A_{0} A_{1} y \leq-c_{d}|y|^{2}+c_{d}^{-1}|B y|^{2}
$$

for all $y \in \mathbb{R}^{n}$

We conclude this section with the following remark:

Remark 1. In studying IBVPs for hyperbolic systems, it is important to distinguish whether the boundary is characteristic or not [21], that is, whether $A_{1}$ is invertible or not. In the above discussions, the invertibility of $A_{1}$ plays the role of the starting point. When $A_{1}$ is not invertible, a modified GKC was proposed in [26]. It covers the above GKC for noncharacteristic problems.

\section{Reduced Boundary Conditions}

Besides the GKC, deriving reduced boundary conditions is another crucial issue in studying the zero relaxation limit for the initial-boundary-value problems. The relaxation limit satisfies the equilibrium system under the structural stability condition [14]. When the problem is given in the half-space, the equilibrium system alone can not determine the limit. It must be supplemented with proper boundary conditions. As part of the system determining the limit, such $\mathrm{BC}$ s should be completely derived from the relaxation system and its BCs. BCs thus derived are called reduced boundary conditions. 
For linearized system (3) satisfying the structural stability condition, we assume without loss of generality that $Q=\operatorname{diag}(0, S)$ with $S$ being a negative definite matrix. Corresponding to the partition of $Q$, we write coefficient matrix $A_{j}$ as

$$
A_{j}=\left(\begin{array}{ll}
A_{j 11} & A_{j 12} \\
A_{j 21} & A_{j 22}
\end{array}\right) .
$$

Thus, the aforementioned equilibrium system reads as

$$
\left\{\begin{array}{l}
v=0, \\
u_{t}+\sum_{j=1}^{d} A_{j 11} u_{x_{j}}=0 .
\end{array}\right.
$$

Recall that coefficient matrices $A_{j 11}$ are symmetrizable.

Let $p_{1}$ be the number of positive eigenvalues for matrix $A_{111}$. According to the classical theory of IBVPs for hyperbolic systems [17,22], equilibrium system (10) needs $p_{1}$ BCs satisfying the UKC to be well-posed. As mentioned before, these BCs should be completely determined from the relaxation system and its BCs:

$$
B U(0, \hat{x}, t)=\left(B_{u}, B_{v}\right)\left(\begin{array}{l}
u \\
v
\end{array}\right)(0, \hat{x}, t)=b(t) .
$$

In this regard, it was established in $[23,27]$ that

Theorem 1. Under the structural stability condition and the $G K C$, there exists a $p_{1} \times p$-matrix $B_{p}$, unique up to an invertible $p_{1} \times p_{1}$-matrix multiplying $B_{p}$ from left, such that relation

$$
B_{p} B_{u} u(0, \hat{x}, t)=B_{p} b(\hat{x}, t)
$$

satisfies the UKC as a BC for the equilibrium system. Moreover, if boundary $x_{1}=0$ is characteristic for the equilibrium system, $B C$ (12) does not involve the characteristic modes corresponding to the zero eigenvalue.

The equilibrium system with the reduced BC (12) consist of a well-posed IBVP since the reduced $B C$ satisfies the UKC. The proof of this theorem involves the perturbation theory of linear operators [28] and subtle matrix analysis. The key is to analyze the limit of right-stable matrix $R_{M}^{S}(\xi, \omega, \eta)$ as $\eta \rightarrow \infty$. According to the $G K C, B R_{M}^{S}(\xi, \omega,+\infty)$ is a $p \times p$ invertible matrix. Then, $B_{p}$ can be chosen as the $p_{1} \times p$-matrix consisting of the first $p_{1}$ rows of $\left[B R_{M}^{S}(\xi, \omega,+\infty)\right]^{-1}$. Such a matrix $B_{p}$ is independent of $\xi$ and $\omega$. We omit the details here, and the interested reader is referred to to $[23,27]$.

Regarding this theorem:

\section{Remark 2.}

- $\quad$ For nonlinear problems, reduced BCs are quite complicated, but can be derived by considering boundary-layer equations

$$
A_{1}(U) U_{y}=Q(U), \quad y=\frac{x_{1}}{\epsilon} \geq 0
$$

For the details, the interested reader is referred to [23].

- In case the boundary is characteristic (for the relaxation system), a similar result can be found in [26]. 
To see the validity of the reduced BCs, we consider asymptotic solutions of the form:

$$
\begin{aligned}
\left(\begin{array}{c}
u_{\epsilon} \\
v_{\epsilon}
\end{array}\right)\left(x_{1}, \hat{x}, t ; \epsilon\right)= & \left(\begin{array}{c}
\bar{u} \\
\bar{v}
\end{array}\right)\left(x_{1}, \hat{x}, t\right)+\left(\begin{array}{c}
\mu_{0} \\
v_{0}
\end{array}\right)\left(\frac{x_{1}}{\epsilon}, \hat{x}, t\right) \\
& +\left(\begin{array}{c}
\mu_{1} \\
v_{1}
\end{array}\right)\left(\frac{x_{1}}{\sqrt{\epsilon}}, \hat{x}, t\right)+\sqrt{\epsilon}\left(\begin{array}{c}
\mu_{2} \\
v_{2}
\end{array}\right)\left(\frac{x_{1}}{\sqrt{\epsilon}}, \hat{x}, t\right)
\end{aligned}
$$

for relaxation system (5) and its BC (11). Here, $(\bar{u}, \bar{v})$ is the outer solution, and $\left(\mu_{i}, v_{i}\right)$ $(i=0,1,2)$ are boundary-layer corrections satisfying matching conditions

$$
\left(\mu_{i}, v_{i}\right)(\infty, \hat{x}, t)=0, \quad i=0,1,2 .
$$

By a standard procedure [27], the outer solution solves equilibrium system (10) with reduced $B C$ (12), while the other terms in (13) can also be determined.

If the boundary is not characteristic for the equilibrium system, there is no boundarylayer with length $\sqrt{\epsilon}$. In other words, the last two terms in (13) are void, and the three scales in the expansion degenerate to two scales.

The validity of the reduced BC (12) is verified with the following theorem.

Theorem 2 ([27]). Assuming that the structural stability condition and the GKC hold, the initial data are given at the equilibrium, and the $B C$ is compatible with the initial condition, there exists a constant $K>0$, such that the exact solution $\left(u^{\varepsilon}, v^{\varepsilon}\right)$ fulfils the following estimate.

$$
\left\|\left(u^{\epsilon}-u_{\epsilon}, v^{\epsilon}-v_{\epsilon}\right)(\cdot, \cdot, t)\right\|_{L^{2}\left(\mathbb{R}^{+} \times \mathbb{R}^{d-1}\right)} \leq K \epsilon^{1 / 2}
$$

for all time $t \in[0, T]$.

Next, we return to one-dimensional linearized moment closure system (9) to illustrate the reduced BCs. For this model, we have

$$
A_{1}=\left(\begin{array}{cc}
A_{11} & A_{12} \\
A_{21} & A_{22}
\end{array}\right)=\left(\begin{array}{ccc|c}
0 & \rho_{0} & 0 & 0 \\
\theta_{0} / \rho_{0} & 0 & 1 & 0 \\
0 & 2 \theta_{0} & 0 & 6 / \rho_{0} \\
\hline 0 & 0 & \rho_{0} \theta_{0} / 2 & 0
\end{array}\right)
$$

and

$$
B=\left(B_{u}, B_{v}\right)=\left(\begin{array}{ccc|c}
0 & 1 & 0 & 0 \\
0 & 0 & \chi \rho_{0} \sqrt{\theta_{0}} & -1
\end{array}\right), \quad b(t)=0 .
$$

As shown in [25], coefficient matrix $A_{1}$ has two positive eigenvalues, $\sqrt{3 \pm \sqrt{6}} \sqrt{\theta_{0}}$, and $A_{11}$ has one positive eigenvalue $\sqrt{3 \theta_{0}}$, that is, $p=2$ and $p_{1}=1$. Thus equilibrium system

$$
\left(\begin{array}{c}
\bar{\rho} \\
\bar{u} \\
\bar{\theta}
\end{array}\right)_{t}+\left(\begin{array}{ccc}
0 & \rho_{0} & 0 \\
\theta_{0} / \rho_{0} & 0 & 1 \\
0 & 2 \theta_{0} & 0
\end{array}\right)\left(\begin{array}{c}
\bar{\rho} \\
\bar{u} \\
\bar{\theta}
\end{array}\right)_{x_{1}}=0
$$

needs one reduced boundary condition. On the other hand, in this case, we have

$$
B R_{M}^{S}(\xi,+\infty)=\left(\begin{array}{cc}
\sqrt{3 \theta_{0}} / \rho_{0} & 0 \\
2 \chi \theta_{0} \sqrt{\theta_{0}} & -\chi \theta_{0} \sqrt{\theta_{0}}
\end{array}\right)
$$


This matrix is obviously invertible, and its inverse is a lower-triangular matrix. Therefore, $B_{p}$ can be chosen as the $1 \times 2$-matrix $(1,0)$, and the reduced $B C$ is

$$
B_{p} B \bar{U}(0, t)=(1,0)\left(\begin{array}{cccc}
0 & 1 & 0 & 0 \\
0 & 0 & \chi \rho_{0} \sqrt{\theta_{0}} & -1
\end{array}\right) \bar{U}(0, t)=B_{p} b(t)=0
$$

with $\bar{U}=(\bar{\rho}, \bar{u}, \bar{\theta}, \bar{f})$. That is, just no-slip velocity BC

$$
\bar{u}(0, t)=0 .
$$

\section{Construction of BCs}

In this section, we show how the theory presented above can be used to construct BCs for PDEs (2). Usually, the relaxation system is derived by augmenting certain classical equations to take account into more refined physical processes. However, proper BCs for the relaxation systems are not always available, since the physical meaning of the nonequilibrium variables is often unclear (e.g., the higher-order moments in momentclosure systems $[6,16])$. Because of this, no physical means can be expected to obtain the $\mathrm{BC}$ s for certain relaxation systems.

For our construction of the $\mathrm{BCs}$, we assume that both the relaxation systems and the well-posed BCs for the corresponding equilibrium systems are given. This assumption is reasonable because there already exist many well-known approaches to construct the relaxation systems, and much knowledge on equilibrium systems and their BCs exists.

We illustrate the construction with the following simple model.

$$
\begin{aligned}
\partial_{t} u-\partial_{x} v & =0, \\
\partial_{t} v-\partial_{x} \sigma & =0, \\
\partial_{t}(\sigma-E u) & =(g(u)-\sigma) / \epsilon .
\end{aligned}
$$

This model was proposed in [29] for the isothermal motions of a viscoelastic material, and can be viewed as an augmentation of classical equations of isothermal elastodynamics:

$$
\begin{aligned}
\partial_{t} u-\partial_{x} v & =0, \\
\partial_{t} v-\partial_{x} g(u) & =0 .
\end{aligned}
$$

Here, $u$ and $\sigma$ denote strain and stress, $v$ is related to particle velocity, $E$ is a positive constant called dynamic Young's modulus, $\epsilon$ is the relaxation time, and $g(u)$ is a given function of $u$. For this model, the structural stability condition reads as

$$
0<g^{\prime}(u)<E .
$$

For simplicity, we assume that the model was defined in the domain where $x \geq-\alpha t$ and $g(u)=G u$, with $G>0$ a constant and $\alpha$ a parameter. Under change in variables $(x, t) \rightarrow(x+\alpha t, t)$, the system (14) becomes

$$
\left(\begin{array}{l}
u \\
v \\
p
\end{array}\right)_{t}+\left(\begin{array}{ccc}
\alpha & -1 & 0 \\
-G & \alpha & -1 \\
0 & G-E & \alpha
\end{array}\right)\left(\begin{array}{l}
u \\
v \\
p
\end{array}\right)_{x}=\frac{1}{\epsilon}\left(\begin{array}{ccc}
0 & 0 & 0 \\
0 & 0 & 0 \\
0 & 0 & -1
\end{array}\right)\left(\begin{array}{l}
u \\
v \\
p
\end{array}\right)
$$

with $p=\sigma-G u$ and (15) reads as

$$
\left(\begin{array}{l}
u \\
v
\end{array}\right)_{t}+\left(\begin{array}{cc}
\alpha & -1 \\
-G & \alpha
\end{array}\right)\left(\begin{array}{l}
u \\
v
\end{array}\right)_{x}=0
$$


Moreover, the given $\mathrm{BC}$ for the equilibrium system is

$$
\hat{B}\left(\begin{array}{c}
u \\
v
\end{array}\right)(0, t)=\hat{b}(t)
$$

with $\hat{B}=\left(\hat{B_{1}}, \hat{B_{2}}\right)$ being a constant full-rank matrix. The number of rows of $\hat{B}$ is equal to the number of positive eigenvalues of coefficient matrix

$$
A_{11} \equiv\left(\begin{array}{cc}
\alpha & -1 \\
-G & \alpha
\end{array}\right)
$$

This matrix has eigenvalues $\alpha \pm \sqrt{G}$, while coefficient matrix

$$
A_{1} \equiv\left(\begin{array}{ccc}
\alpha & -1 & 0 \\
-G & \alpha & -1 \\
0 & G-E & \alpha
\end{array}\right)
$$

has eigenvalues $\alpha \pm \sqrt{E}$ and $\alpha$.

To be precise and simple, we chose $\alpha \in(-\sqrt{E},-\sqrt{G})$, while other choices can be found in [30]. For such an $\alpha$, boundary $x=0$ is noncharacteristic for both (17) and (16), boundary matrix $\hat{B}$ is void, and we need to construct one $B C$ of the form

$$
B\left(\begin{array}{c}
u(0, t) \\
v(0, t) \\
p(0, t)
\end{array}\right)=b_{\epsilon}(t)
$$

for the relaxation system (16).

To find such a nonzero vector $B \in \mathbb{R}^{3}$, we consider the asymptotic solution of form

$$
\left(\begin{array}{c}
u_{\epsilon} \\
v_{\epsilon} \\
p_{\epsilon}
\end{array}\right)(x, t ; \epsilon)=\left(\begin{array}{c}
\bar{u} \\
\bar{v} \\
\bar{p}
\end{array}\right)(x, t)+\left(\begin{array}{c}
\mu \\
v \\
\pi
\end{array}\right)\left(\frac{x}{\epsilon}, t\right)
$$

and require it to satisfy the $\mathrm{BC}(18)$ :

$$
B\left(\begin{array}{c}
\bar{u}+\mu \\
\bar{v}+v \\
\bar{p}+\pi
\end{array}\right)(0, t)=b_{0}(t) .
$$

In this way, we obtain some algebraic relations or restrictions on $B$, from which we deduce that

$$
B^{*}=\left(\begin{array}{c}
1+\frac{C_{1}}{\alpha^{2}-G} \\
\sqrt{G}+\frac{\alpha C_{1}}{\alpha^{2}-G} \\
C_{1}
\end{array}\right) \times\left(\begin{array}{c}
1+\frac{C_{2}}{\alpha^{2}-G} \\
-\sqrt{G}+\frac{\alpha C_{2}}{\alpha^{2}-G} \\
C_{2}
\end{array}\right)
$$

with $C_{1}, C_{2}$ two free parameters. BCs thus constructed are not unique, which is expected.

It was shown in [30] that, when $C_{1}, C_{2}$ are both sufficiently small, the constructed $\mathrm{BC}$ are strictly dissipative. Thus, they satisfy the GKC. On this basis, the validity of the constructed BCs was rigorously proved in [30].

\section{Concluding Remarks}

In this paper, we presented a systematical review on boundary conditions (BCs) for partial differential equations (PDEs) from nonequilibrium thermodynamics. From a stabil- 
ity point of view, such PDEs should satisfy the structural stability condition. In particular, they constitute hyperbolic systems, for which the uniform Kreiss condition (UKC) is a sufficient and essentially necessary condition for the well-posedness of the corresponding models (PDEs with BCs). Taking a linearized moment closure system as an example, we showed that the structural stability condition and UKC do not automatically guarantee the compatibility of the models with the corresponding classical models. This motivated the generalized Kreiss condition (GKC) - a strengthened version of the UKC. It is expected that the GKC and the structural stability condition are the very criteria to verify the four fundamental requirements when modeling nonequilibrium phenomena in spatial domains with boundaries.

The compatibility and long-time tendency requirements suggest to study the zero relaxation limit of the PDEs with BCs. It is trivial to obtain the equilibrium system for the limit. However, the equilibrium system alone cannot determine the limit, and proper BCs are needed. The needed BCs are referred to as reduced BCs, and they should be completely determined by the PDEs with the given BCs. The derivation of the reduced BCs is by no means trivial.

Under the GKC and the structural stability condition, we showed how to derive the reduced BCs for general relaxation models. For linearized problems, the validity of the reduced BCs was rigorously verified. For nonlinear problems, such a verification is open. Furthermore, we used a simple example to show how the thus far developed theory can be used to construct proper BCs accompanying PDEs modeling nonequilibrium phenomena in spatial domains with boundaries.

Author Contributions: Conceptualization, W.-A.Y.; investigation, W.-A.Y., Y.Z.; writing-original draft preparation, Y.Z.; writing-review and editing, W.-A.Y., Y.Z.; project administration, W.-A.Y.; funding acquisition, W.-A.Y. All authors have read and agreed to the published version of the manuscript.

Funding: This research was funded by the National Natural Science Foundation of China (grant number 12071246).

Conflicts of Interest: The authors declare no conflict of interest.

\section{References}

1. Van, P. Nonequilibrium thermodynamics: Emergent and fundamental. Philos. Trans. R. Soc. A 2020, 378, 20200066. [CrossRef] [PubMed]

2. de Groot, S.R.; Mazur, P. Non-Equilibrium Thermodynamics; North-Holland Publishing Company: Amsterdam, The Netherlands, 1962.

3. Hyon, Y.; Kwak, D.Y.; Liu, C. Energetic variational approach in complex fluids: Maximum dissipation principle. Discret. Contin. Dyn. Syst. 2017, 26, 1291-1304. [CrossRef]

4. Jou, D.; Casas-Vázquez, J.; Lebon, G. Extended Irreversible Thermodynamics, 4th ed.; Springer: New York, NY, USA, 2010.

5. Lebon, G.; Jou, D.; Casas-Vázquez, J. Understanding Non-Equilibrium Thermodynamics: Foundations, Applications; Springer: London, UK, 2008.

6. Müller, I.; Ruggeri, T. Rational Extended Thermodynamics; Springer: New York, NY, USA, 1998.

7. Öttinger, H.C. Beyond Equilibrium Thermodynamics; Wiley-Interscience: Hoboken, NJ, USA, 2005.

8. Zhu, Y.; Hong, L.; Yang, Z.; Yong, W.-A. Conservation-dissipation formalism of irreversible thermodynamics. J. Non-Equilib. Thermodyn. 2015, 40, 67-74. [CrossRef]

9. Yong, W.-A. Intrinsic properties of conservation-dissipation formalism of irreversible thermodynamics. Philos. Trans. R. Soc. A 2020, 378, 20190177. [CrossRef] [PubMed]

10. Pavelka, M.; Klika, V.; Grmela, M. Multiscale Thermo-Dynamics; de Gruyter: Berlin, Germany, 2018.

11. Kreiss, H.-O.; Lorenz, J. Initial-boundary value problems and the Navier-Stokes equations. In Pure and Applied Mathematics; Academic Press, Inc.: Boston, MA, USA, 1989; Volume 136, p. xii+402.

12. Szücs, M.; Kovács, R.; Simic, S. Open Mathematical Aspects of Continuum Thermodynamics: Hyperbolicity, Boundaries and Nonlinearities. Symmetry 2020, 12, 1469. [CrossRef]

13. Yong, W.-A. Singular Perturbations of First-Order Hyperbolic Systems. PhD Thesis, Universität Heidelberg, Heidelberg, Germany, 1992.

14. Yong, W.-A. Singular perturbations of first-order hyperbolic systems with stiff source terms. J. Differ. Equ. 1999, 155, 89-132. [CrossRef] 
15. Yang, Z.; Yong, W.-A. Validity of the Chapman-Enskog expansion for a class of hyperbolic relaxation systems. J. Differ. Equ. 2015, 258, 2745-2766. [CrossRef]

16. Cai, Z.; Fan, Y.; Li, R. Globally hyperbolic regularization of Grad's moment system in one dimensional space. Commun. Math. Sci. 2013, 11, 547-571. [CrossRef]

17. Benzoni-Gavage, S.; Serre, D. Multidimensional Hyperbolic Partial Differential Equations: First Order Systems and Applications; Clarendon Press: Oxford, UK, 2007.

18. Kreiss, H.-O. Initial boundary value problems for hyperbolic systems. Comm. Pure Appl. Math. 1970, 23, 277-298. [CrossRef]

19. Yong, W.-A. An interesting class of partial differential equations. J. Math. Phys. 2008, 49, 033503. [CrossRef]

20. Yong, W.-A. Basic aspects of hyperbolic relaxation systems. In Advances in the Theory of Shock Waves; Freistühler H., Szepessy, A., Eds.; Progress in Nonlinear Differential Equations and Their Applications; Birkhäuser: Boston, MA, USA, 2001; Volume 47, pp. 259-305.

21. Majda, A.; Osher, S. Initial-boundary value problems for hyperbolic equations with uniformly characteristic boundary. Comm. Pure Appl. Math. 1975, 28, 607-675. [CrossRef]

22. Gustafsson, B.; Kreiss, H.O.; Oliger, J. Time-Dependent Problems and Difference Methods, 2nd ed.; John Wiley \& Sons, Inc.: Hoboken, NJ, USA, 2013.

23. Yong, W.-A. Boundary conditions for hyperbolic systems with stiff source terms. Indiana Univ.Math. J. 1999, 48, 115-137. [CrossRef]

24. Hersh, R. Mixed problems in several variables. J. Math. Mech. 1963, 12, 317-334.

25. Zhao, W.; Yong, W.-A. Boundary conditions for kinetic theory based models II: A linearized moment system. Math. Methods Appl. Sci. 2021. accepted. [CrossRef]

26. Zhou, Y.; Yong, W.-A. Boundary conditions for hyperbolic relaxation systems with characteristic boundaries of type I. J. Differ. Equ. 2021, 281, 289-332. [CrossRef]

27. Zhou, Y.; Yong, W.-A. Boundary conditions for hyperbolic relaxation systems with characteristic boundaries of type II. submitted.

28. Kato, T. A Short Introduction to Perturbation Theory for Linear Operators; Springer: New York, NY, USA, 1982.

29. Făciu, C.; Mihăilescu-Suliciu, M. The energy in one-dimensional rate-type semilinear viscoelasticity. Int. J. Solids Struct. 1987, 23, 1505-1520. [CrossRef]

30. Zhou, Y.; Yong, W.-A. Construction of boundary conditions for hyperbolic relaxation approximations I: The lin-earized Suliciu model. Math. Model. Methods Appl. Sci. 2020, 30, 1407-1439. [CrossRef] 